\title{
Implementing Optical Phased Array Beam Steering with Cascaded Microlens Arrays"
}

\author{
Edward. A. Watson \\ Air Force Research Laboratory, Electro-Optical Technology Division \\ AFRL/SNJ BIdg 620 \\ 2241 Avionics Circle \\ Wright-Patterson AFB, OH 45433-7304 \\ 937-255-4039 X4030 \\ Edward. Watson(owpatb.af.nil \\ Walter E. Whitaker III \\ OMI \\ AFRL/SNJM Bldg 622 \\ 3109 P Street \\ Wright-Patterson AFB, OH 45433-7700 \\ 937-255-9614 X237 \\ Walter.Whitaker@wpafb.af.mil \\ Christopher D. Brewer \\ Electro-Optics Department, University of Dayton \\ AFRL/SNJM Bldg 622 \\ 3109 P Street \\ Wright-Patterson AFB, OH 45433-7700 \\ 937-255-9614 X292 \\ Christopher.Brewer@wpafb.af.mil \\ Scott R. Harris \\ Air Force Research Laboratory, Electro-Optical Technology Division \\ AFRL/SNJM Bldg 622 \\ 3109 P Street \\ Wright-Patterson AFB, OH 45433-7700 \\ 937-255-9614 X285 \\ Scott.Harris@wpafb.af.mil
}

\begin{abstract}
A cascade of microlens arrays that are decentered with respect to each other is one potential method for beam steering; the magnitude of the steering angle depends on the amount of decenter. A simple argument is presented that shows that the output of such a system is analogous to the output of an optical phased array. The periodic nature of the exiting wavefront restricts allowed steering angles to values that are determined by the grating equation and the pitch of the microlens arrays. The efficiency of steering into a desired diffraction order of the phased array is determined by the amount of decenter of the microlens arrays, the coherence of the arrays, and the fill-factor at the output of the array. Maximum fill-factor is desired, which can be achieved through the addition of an intermediate microlens array that acts like a field lens. An experimental implementation of such a triplet cascade of microlens arrays, suitable for steering short wave infrared
\end{abstract}

\footnotetext{
* US Government work not protected by US copyright.
}

laser light, is described. The extension of such a cascade to steering broad spectral band light is also discussed.

\section{TABLE OF CONTENTS}

1. INTRODUCTION

2. BEAM SteERING USING AN ARRAy OF MiCROLENS TRIPLETS

3. MiCROLENS ARRAY CHARACTERIZATION

4. BROAD SPECTRAL BAND BEAM STEERING

5. SUMMARY

\section{INTRODUCTION}

The agile steering of optical beams, such as from a laser, has application to laser radar, optical communications, optical interconnects, and spatial light modulator addressing. Several methods of beam steering, such as 
mechanical motion of mirrors, electro-optic cells, and acousto-optic cells, have been considered in the past ${ }^{1}$ and additional beam steering methods are currently receiving attention. ${ }^{2}$ These methods include dynamic gratings using spatial light modulators ${ }^{3}$ and decentered microlens arrays. ${ }^{4-6}$ The concept of beam steering using decentered microlens arrays (MLAs) is easily understood by considering two macroscopic lenses. A collimated input beam is focused to the back focal point of the first lens. If the back focal plane of the first lens is also the front focal plane of the second lens, then the second lens will re-collimate the light. If the second lens is decentered with respect to the first lens, then the back focal point of the first lens appears as an off-axis point to the second lens, though the point remains in the front focal plane of the second lens. The second lens again collimates the light, but the beam is directed to a non-zero field angle. A simple trace of the principal ray shows that the tangent of the steer angle is equal to the amount of decenter divided by the focal length of the second lens.

The agile steering of a beam using the decentered lens concept requires that the second lens in the pair be moved rapidly. If macroscopic optics are used, large amplitude, rapid motion may be difficult achieve. An alternative to using macroscopic optics is to use microlens arrays. Microlens arrays are lightweight, and because they have small focal lengths (typically on the order of a millimeter or less) ${ }^{4}$, the amount of decenter required to achieve a desired steering angle is also small. Since microlens arrays are periodic, the maximum decenter is restricted to half of a microlens diameter. ${ }^{4}$ Therefore, the maximum steering angle is simply $\theta_{\max }=\arctan [1 /(2 \mathrm{f} / \#)]$. Large angle steering necessarily requires that small f-number microlens arrays be used.

The use of microlens arrays for beam steering, especially with small f-number, requires that several issues be addressed. It has been shown that the periodic structure of the arrays makes their ideal output analogous to that of a blazed diffraction grating. ${ }^{6}$ As is well known, gratings steer light to discrete locations and can steer with good efficiency only if the blaze angle is chosen appropriately. In addition to these grating effects, imperfections in the arrays will also affect the far field irradiance pattern. In Sec. 2 we describe an implementation of microlens beam steering using three cascaded microlens arrays. In Sec. 3 we discuss characterization of the microlens arrays to aid in understanding the results of Sec. 2. In Sec. 4 we consider the effects of the grating-like nature of the arrays on broad spectral band beam steering.

\section{BEAM STEERING USING AN ARRAY OF}

\section{MICROLENS TRIPLETS}

The basic concept of beam steering using microlens arrays is illustrated in Fig. 1. Each set of three microlenses produces a tilted wavefront. From a Fourier optics point of view, the first lens produces an off-axis point source for the last lens. Application of the Fourier Transform Shift Theorem indicates that this shifted point source then produces a wavefront with a linear phase gradient (tilt) after passage through the last lens. The output of the last microlens array is periodic; therefore, the wavefront will have properties similar to those of the wavefront exiting a diffraction grating. These properties include discrete steering angles associated with the period of the grating and chromatic dispersion. While beam steering can be performed using only two microlens arrays, the intermediate microlens array is used to increase the fill factor at the output array. ${ }^{6}$ If the intermediate array is inserted at the focal plane of the input array, it will act as a field lens and keep the chief ray of each ray bundle centered on the corresponding microlens in the output array.

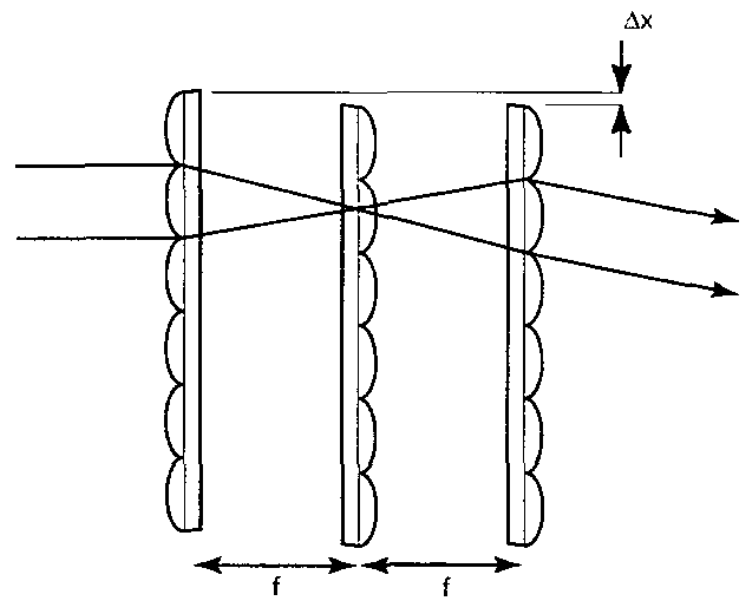

Fig. 1. Diagram of the microlens array beam steering concept.

Under the assumptions that the input to each microlens triplet is a plane-wave and that the diffraction effects of the finite aperture of the first lens can be ignored, the wavefront exiting the third lens can be shown to be ${ }^{6}$

$$
T(x, y) \propto e^{\frac{i 2 \pi}{\lambda f}(x \Delta x+y \Delta y)} \operatorname{rect}\left(\frac{x-\Delta x}{d}, \frac{y-\Delta y}{d}\right),
$$

where $f$ is the focal length of each microlens, $d$ is the diameter of each microlens, and $\Delta x$ and $\Delta y$ describe the amount of decenter in the $x$ and $y$ directions. The linear phase term describes the wavefront tilt and is a function of the decenter. The aperture function described by the rect function is shifted by the decenter values $\Delta x$ and $\Delta y$, and the width of the rect function is equal to the diameter of the microlens, therefore, the fill-factor is unity.

If we now assume that the effect of arrays of microlenses can be represented by a convolution of Eq. (1) with a 
sampling function, then the output after the third microlens array of width $D$ can be written as

$$
\begin{gathered}
T(x, y) \propto e^{\frac{i 2 \pi}{\lambda f}(x \Delta x+y \Delta y)} \operatorname{rect}\left(\frac{x-\Delta x}{d}, \frac{y-\Delta y}{d}\right) \\
\otimes \frac{1}{d^{2}}\left[\operatorname{rect}\left(\frac{x}{D}, \frac{y}{D}\right) \operatorname{comb}\left(\frac{x}{d}, \frac{y}{d}\right)\right]
\end{gathered} .
$$

The resulting far field pattern, E, is obtained by taking the Fourier transform of (2).

$$
\begin{aligned}
E\left(x_{i}, y_{i}\right) & \propto \operatorname{sinc}\left[d\left(\frac{x_{i}}{\lambda r}-\frac{\Delta x}{\lambda f}, \frac{y_{i}}{\lambda r}-\frac{\Delta y}{\lambda f}\right)\right] \\
& \times\left[\operatorname{sinc}\left(\frac{D x_{i}}{\lambda r}, \frac{D y_{i}}{\lambda r}\right) \otimes,\right. \\
& \left.\sum_{n, m} \delta\left(\frac{x_{i}}{\lambda r}-\frac{n}{d}, \frac{y_{i}}{\lambda r}-\frac{m}{d}\right)\right]
\end{aligned}
$$

where $x_{i}$ and $y_{i}$ are coordinates in the observation plane and $r$ is the distance from the beam steering arrays to the observation plane. In Eq. (3) we have explicitly shown the delta functions that make up the comb function from Eq. (2). The locations of the grating orders are given by the locations of the delta functions. The amount of energy in each of the grating modes is determined by the first sinc function. The width of the pattern replicated at each of the grating modes is defined by the second sinc function.

Upon inspection of Eq. (3) we find that if the decenter values are chosen properly, then the zeros of the first sinc function lie on top of all the grating modes except the one on which the sinc is centered. Hence, light is steered only to that order. Note, however, that the available steering angles are still limited to the modes of the grating betermined by the period of the microlenses. Because of the inclusion of the third array, acting as a field lens, it is expected that, to first order, the efficiency of steering should be independent of the steering angle. ${ }^{6}$

In order to evaluate the actual steering efficiency of such a setup, we implemented the triplet microlens array beam steering concept. The specifications of these arrays are shown in Table 1.

\begin{tabular}{|c|c|}
\hline Microlens Width & $0.3 \mathrm{~mm}$ \\
\hline Array Width & $10 \mathrm{~mm}$ \\
\hline Microlens Focal Length & $0.45 \mathrm{~mm}$ \\
\hline Microlens f-number & 1.5 \\
\hline Number of Elements & $32 \times 32$ \\
\hline Design Wavelength & $1064 \mathrm{~nm}$ \\
\hline
\end{tabular}

Table 1. Characteristics of the Microlens Arrays.

For an f-number of 1.5 , the expected maximum steering angle is $+/-18.4^{\circ}$. Measured steering efficiencies are shown in Fig. 2. As can be seen from the figure, the power in the steered spot is reasonably independent of steering position. The half-power steering points are at approximately $+/-17^{\circ}$, in reasonable agreement with the theoretical maximum. The steered power in the positive angular direction was slightly clipped by a mount in the experimental set-up, which explains the asymmetry in the plot. The energy measured in the steered spot was found to be within $3 \%$ of what would be predicted based on Fresnel reflection losses at the 6 surfaces associated with the microlens array triplet.

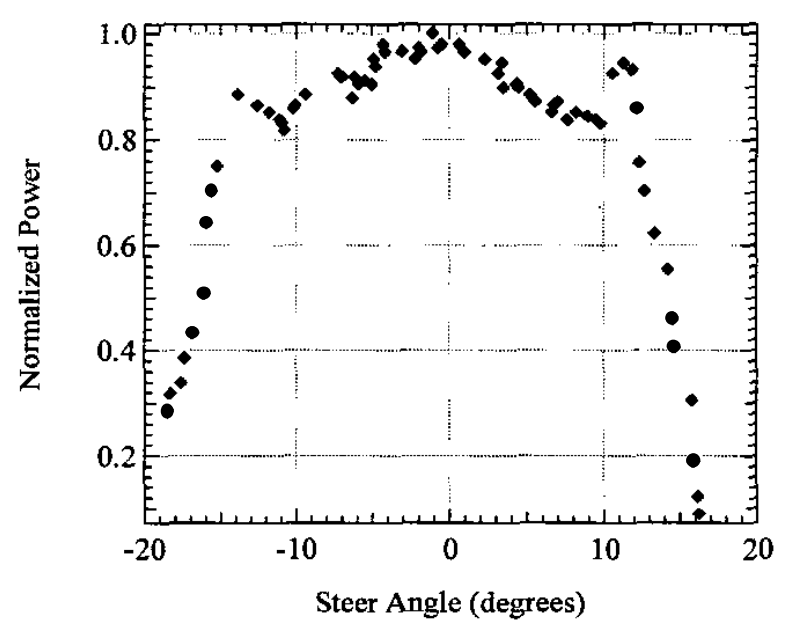

Fig. 2. Power as a function of steering angle.

Figure 3 shows an example far field image of a steered beam. The spot is highly structured and the periodic irregularities in the beam correspond to the order spacing predicted from the grating equation and the lenslet period. Furthermore, the spot diameter was found to be many times larger than what would be expected from a diffractionlimited spot associated with an input beam diameter of 5 $\mathrm{mm}$. The reasons for the spot shape and size are discussed in the next section.

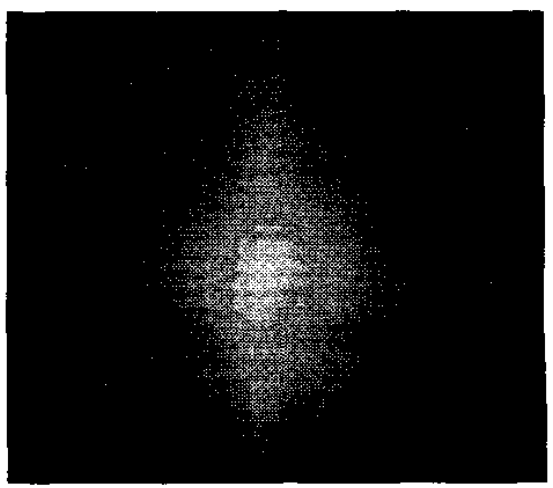


Fig. 3. Far field spot associated with triplet microlens beam steering. This spot does not exhibit the characteristics expected from a diffraction-limited, 5 mm aperture.

\section{MiCROLENS CHARACTERIZATION}

In deriving Eq. (3) it was assumed that each microlens triplet produced identical wavefronts (i.e. the arrays are "coherent"). The resulting far field pattern for a wavefront exiting a coherent array is diffraction limited by the full aperture of the microlens array. If there are random variations in the output of each triplet, then the far field pattern takes on the shape of the diffraction pattern closer to that resulting from a single microlens triplet. Such a microlens array is termed "incoherent." In practice, the resulting far field pattern can be described as a combination of patterns from both coherent and incoherent arrays. ${ }^{7}$ There are many factors that can cause the array to be incoherent. These include variations in thickness of the substrate that result in a varying phase offset for each lenslet, variations in the focal length of each microlens, and variations in the optical axis direction of each lenslet. ${ }^{7}$ For beam steering applications it is desirable that the array be as coherent as possible.

We characterized several micro-lens arrays to determine their coherence properties. The arrays are categorized as either refractive or diffractive, depending on whether the individual microlenses are refractive or diffractive optical elements. The primary advantage of refractive microlens arrays is that the elements themselves can have less chromatic dependence and do not produce the multiple focal points typically associated with diffractive lenses. The primary advantage of diffractive microlens arrays is that the profile of each lenslet can be more repeatable and precisely controlled. This repeatability is important for the microlens array to produce a beam with diffraction properties associated with the full array aperture as opposed to that of a single microlens.

In an effort to quantify the coherence properties of various microlens arrays, we employed the experimental set up shown in Fig. 4 to examine a pair of $\mathrm{f} / 74 \mathrm{MLA}$ 's (DMLA $=$ $0.328 \mathrm{~mm}$ ), two diffractive lenses with $\mathrm{f} / 1.5\left(\mathrm{D}_{\mathrm{MLA}}=0.300\right.$ $\mathrm{mm}$ ), and the refractive lenses of Sec. 2 . Note that the diffractive and refractive arrays were designed for a wavelength of $\lambda=1.064 \mu \mathrm{m}$ while the $\mathrm{f} / 74$ lenslets were designed for $\lambda=633 \mathrm{~nm}$. The beam propagates through a polarizer $(\mathrm{P})$ and a half-wave plate $(\lambda / 2)$ before illuminating a polarizing beam splitter cube (PBSC). Adjusting the polarization to ensure maximum reflection from the PBSC, the beam is rotated $45^{\circ}$ with a quarter-wave plate $(\lambda / 4)$ before transmission through a MLA mounted exactly one focal length away from reflective mirror (M). Each lenslet then focuses to a spot on the mirror surface (M). The reflected beam is re-collimated back through the array. Thus the beam passes through the array twice and experiences the same phase distortion twice. The output of the autocollimation is rotated another $45^{\circ}$ for maximum transmission through the PSBC. Lenses $\mathrm{L}_{1}$ and $\mathrm{L}_{2}$ then form a telescope to ease the alignment of the micro-lens array and the magnified image is brought to a focus on a Kodak MegaPlus camera one focal length from $\mathrm{L}_{3}$.

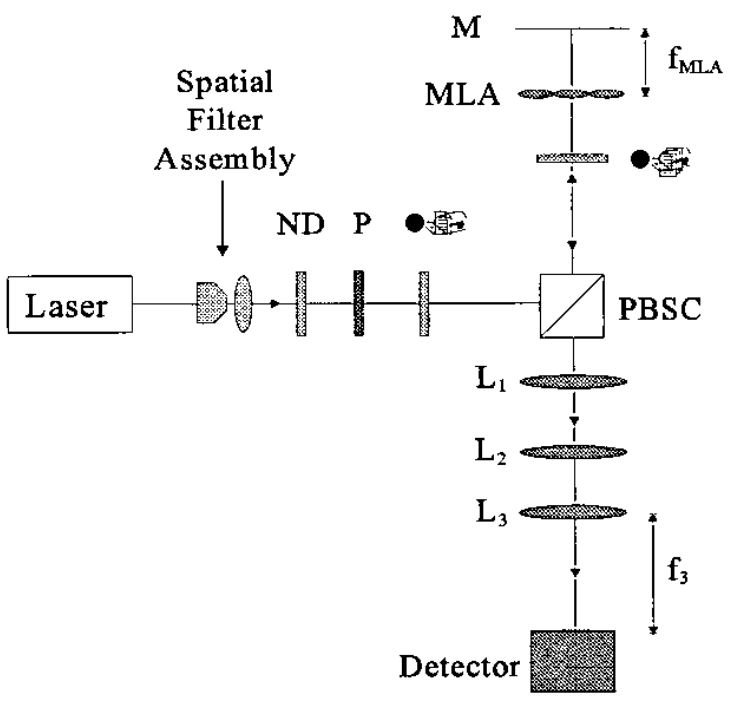

Fig. 4. Experimental setup used to analyze the coherence properties of a single microlens array.

The microlens arrays were illuminated with a $5 \mathrm{~mm}$ diameter beam so that the irradiance pattern at the camera was the result of the statistical wavefront variations imposed by approximately 200 individual lenslets. Presuming the microlens arrays are at least partially coherent, the expected irradiance pattern $I$ in the focal plane can be described as a linear combination of coherent $\mathrm{I}_{\text {coherent }}$ and incoherent $\mathrm{I}_{\text {incoherent }}$ parts and is written as

$$
\mathrm{I}=\kappa \mathrm{I}_{\text {coherent }}+(1-\kappa) \mathrm{I}_{\text {incoherent }}
$$

where $\kappa$ is a measure of the relative coherence of the array. The irradiance patterns $\mathrm{I}_{\text {cohereat }}$ and $\mathrm{I}_{\text {incoherent }}$ are the far field patterns (sinc functions) due to square apertures associated with the full array or a single microlens. We used the method developed by Glöckner and Göring ${ }^{7}$ to determine $\kappa$ from measurements of the encircled intensity in a focal spot as a function of radius. By varying the value of $K$ in the theoretical expression for I, the RMS error between the data and theory can be minimized, resulting in an estimated value for the coherence parameter, $\kappa$. This process is repeated 20 times at different locations on the microlens array and the average $\kappa$ is computed. Table 2 gives the estimate of $\kappa$ and standard deviation for each of the different microlens arrays examined.

As can be seen, the measured coherence parameter, even between MLAs of the same type, varies significantly. The 
low $\mathrm{f}$-number refractive arrays have the lowest coherence factor. Therefore, it is not surprising that the spot diameter resulting from their use in the previous section does not correspond to the diffraction limited spot associated with the input beam. We find in general that the array coherence for the diffractive lenslets is higher than the $f / 74$ and the f/1.5 refractive arrays. Unfortunately, with the data taken to date, this trend cannot be attributed to any single statistical variation in the focal length, substrate thickness, or optical axis tilt.

\begin{tabular}{|c|c|c|c|c|}
\hline Array & $\kappa(633 \mathrm{~nm})$ & $\sigma_{\kappa}$ & $\kappa(1 \mu \mathrm{m})$ & $\sigma_{\kappa}$ \\
\hline $\begin{array}{c}\text { Refractive } \\
\mathrm{f} / 74, \# 1\end{array}$ & 0.744 & 0.037 & NA & NA \\
\hline $\begin{array}{c}\text { Refractive } \\
\mathrm{f} / 74, \# 2\end{array}$ & 0.865 & 0.037 & NA & NA \\
\hline $\begin{array}{c}\text { Diffractive } \\
\text { f/1.5, \#1 }\end{array}$ & 0.921 & 0.015 & 0.893 & 0.033 \\
\hline $\begin{array}{c}\text { Diffractive } \\
\text { f/1.5, \#2 }\end{array}$ & 0.805 & 0.018 & 0.754 & 0.042 \\
\hline $\begin{array}{c}\text { Refractive } \\
\text { f/1.5, \#1 }\end{array}$ & 0.463 & 0.035 & 0.696 & 0.057 \\
\hline $\begin{array}{c}\text { Refractive } \\
\text { f } / 1.5, \# 2\end{array}$ & 0.379 & 0.055 & 0.550 & 0.058 \\
\hline
\end{tabular}

Table 2: Summary of individual coherence parameters for various micro-lens arrays measured using the autocollimation method. $\sigma_{\mathrm{\kappa}}$ is the standard deviation of each measurement.

Because a beam steering system uses more than one microlens array, we investigated the coherence effect when multiple cascaded arrays were used in place of a single array in the double pass configuration. The experimental setup for this case is shown in Fig. 5. The configuration is similar to that in Fig. 4 except that the autocollimation through a single array is replaced with a 4-f system using two arrays.

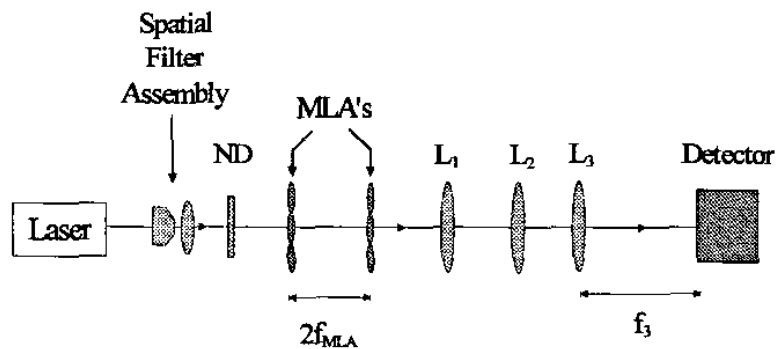

Fig. 5. Experimental setup used to analyze the coherence properties of two cascaded micro-lens arrays.

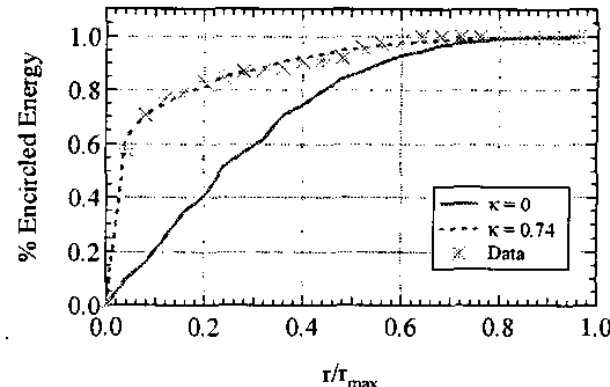

(a)

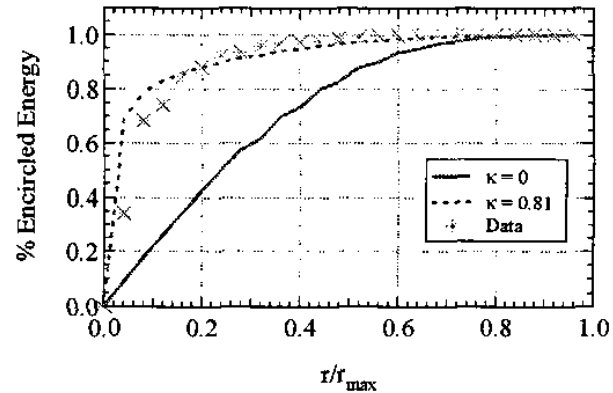

(b)

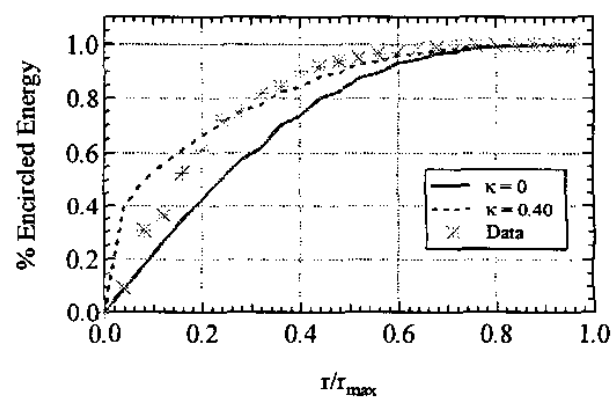

(c)

Fig. 6. Example encircled energy plots used to determine the coherence factor of the cascaded (a) refractive microlens arrays with $f / 74$, (b) the diffractive microlens arrays with $\mathrm{f} / 1.5$ and $(\mathrm{c})$ the refractive microlens arrays with $\mathrm{f} / 1.5$.

Figures 6 (a), (b), and (c) show several encircled energy plots associated with the cascaded microlens array measurements. The dashed line represents the theoretical curve associated with the $\kappa$ value that gave the best fit. The solid line represents the encircled energy plot expected for an array with a coherence factor of $\kappa=0$.

Data like those in Fig. 6 were then used to compute the coherence values and standard deviations for pairs of each type of microlens array listed in Table. 3. The average values of $\kappa$ associated with each pair of MLA's from Table 2 are also given for comparison.

Examining the coherence parameter for the $f / 74$ and $f / 1.5$ refractive lenslets, we find $\kappa$ is not dominated by a single array, but is an average of the two arrays. By passing through each micro-lens only once, the phase deviation 
introduced by the second array statistically cancels out some of the effects of the first array. Therefore even if one array in the system is particularly bad, its overall contribution to the image degradation is much lower than in the autocollimation system. One also notices that increasing the source wavelength from $\lambda=633 \mathrm{~nm}$ to the design wavelength of $1.064 \mu \mathrm{m}$, the array coherence factor for both the diffractive and refractive f/1.5 lenslets is enhanced as well. In the case of the refractive $f / 1.5$ arrays, each lenslet has an aspheric figure optimized for $1.064 \mu \mathrm{m}$, while the diffractive arrays were simply designed to operate at 1.064 $\mu \mathrm{m}$. Subsequentiy, more energy is located towards the center of the spot pattern, which in turn translates to a higher $\kappa$ value.

\begin{tabular}{|c|c|c|c|c|}
\hline Array & $\lambda(\mu \mathrm{m})$ & $\kappa$ (meas) & $\sigma_{\kappa}$ & $\kappa($ ave $)$ \\
\hline Refractive f/74 & 0.633 & 0.788 & 0.045 & 0.805 \\
\hline Diffractive f/1.5 & 0.633 & 0.812 & 0.066 & 0.863 \\
\hline Diffractive f/1.5 & 1.064 & 0.917 & 0.035 & 0.824 \\
\hline Refractive f/1.5 & 0.633 & 0.466 & 0.059 & 0.421 \\
\hline Refractive f/1.5 & 1.064 & 0.627 & 0.065 & 0.623 \\
\hline
\end{tabular}

Table 3. Comparison of coherence parameters for a set of cascaded micro-lens arrays. Here $\kappa$ was measured directly for the two-array system and the average value calculated from the individual coherence parameters in Table 2.

\section{BRoAd SPECTRAL BAND BEAM STEERING}

Because the microlens array beam steering concept operates in a fashion that is analogous to that of a blazed diffraction grating, it suffers from the same dispersion problems: different wavelengths are steered in different directions. We have investigated a simple concept that can reduce the effects of dispersion by using higher order diffraction peaks. 8

Any periodic optical array operating at normal incidence has diffraction orders that are given by the grating equation

$$
\sin (\theta)=\frac{m \lambda}{d}
$$

where $\mathrm{m}$ is the order number and $d$ is the period of the grating or lenslet array. In order to maximize the amount of energy in a particular order, the structure is blazed by imparting a phase tilt that corresponds to the angle of the desired order over each sub-aperture. Furthermore, the grating period $d$ also determines the angular selectivity of the grating structure. The salient term from Eq. (3) is squared to give

$$
I \propto \operatorname{sinc} 2\left(d \frac{\theta}{\lambda}\right)
$$

Simply put, increasing $d$ narrows the angular selectivity of the grating structure according to $\mathrm{Eq}$, (6). However, this also has the effect of reducing the steer angle in Eq. (5) unless the grating is blazed for a proportionally higher diffraction order.

Figure 7(a) shows steering to the first diffraction order for wavelengths of $1 \mu \mathrm{m}$ and $1.7 \mu \mathrm{m}$. The enveloping curves show the efficiency functions associated with each wavelength. As can be seen, the orders of the different wavelengths are in different locations, although occasionally different orders of the two wavelengths may actually overlap in space. Figure $7(\mathrm{~b})$ shows steering to the second order of the grating. The period of the grating was doubled so that the steering angle is same between the two figures. That is, the second order in Fig. $7(b)$ is in the same location in space as the first order in Fig. 7(a). This matching of the order locations in space results in the other orders of the grating in Fig. 7(b) being in different locations than in Fig. 7(a). It is also apparent that the sinc envelope functions are narrower so that the angular dispersion of in Fig. 7(b) is less than that in 7(a).

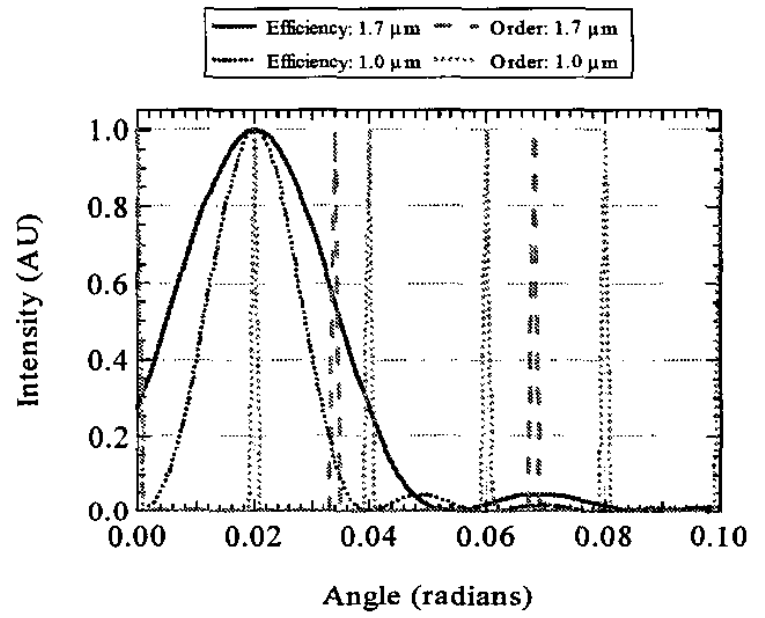

(a)

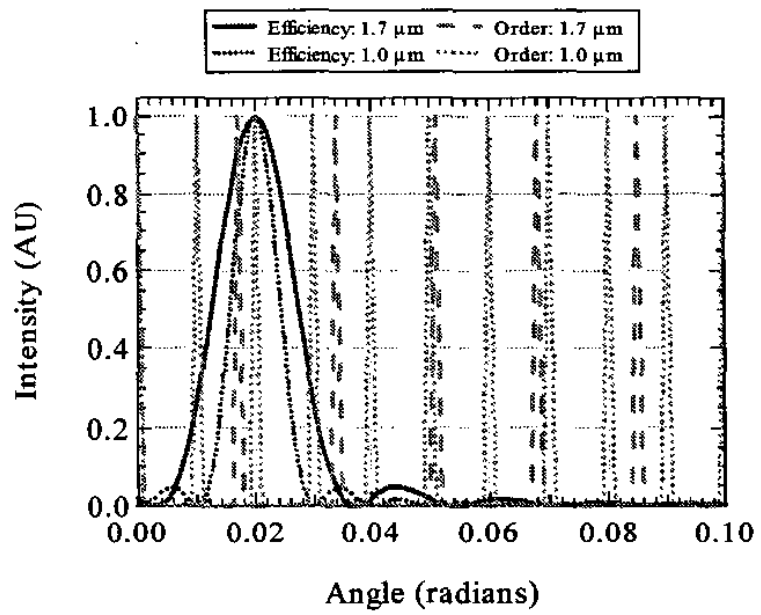

(b)

Fig. 7. Plots of allowed far field steering locations for two different wavelengths. (a). Steering blazed to the first 
grating order. (b). Steering blazed to the second grating order.

This approach may be well suited to a microlens beam steering implementation because the microlens concept steers light by varying the blaze angle of the wavefront exiting the arrays so that the electric fields efficiently constructively interfere at the higher diffraction orders. By increasing the size of the microlenses and steering to higher diffraction orders, reduced dispersion may result. However, larger microlenses may make it more difficult to implement the concept. This tradeoff will require further investigation.

\section{SUMMARY}

We have discussed an approach to agile beam steering that can be used for large angle steering. Decentered microlens arrays have the advantage of requiring small motions to produce large angle steering and can potentially steer with good efficiency. We have found that the coherence properties of the arrays, particularly refractive arrays of small f-number elements, significantly affect the beam pattern in the observation plane, though the steering overall steering efficiency does not appear to be dramatically affected.

\section{REFERENCES}

1. J. D. Zook, "Light Beam Deflector Performance: a Comparative Analysis," Appl. Opt. 13(4), 875-887 (1974).

2. See, for example, the papers in the sessions on Novel Beam-Steering Technology, in Optical Scanning Systems: Design and Applications, L. Beiser and S. F. Sagan, eds., Proc. SPIE 3131 (1997), and in Optical Scanning: Design and Application, L. Beiser, S. F. Sagan, and G. F. Marshall, eds., Proc. SPIE 3787 (1999).

3. T. A. Dorschner, R. C. Sharp, D. P. Resler, L. J. Friedman, and D. C. Hobbs, Basic Laser Beam Agility Techniques (BATS), Final Report, WL-TR-93-1020 (1993).

4. W. Goltsos and M. Holz, "Agile beam steering using binary optics microlens arrays," Opt. Eng. 29(11), 13921397 (1990).

5. K. M. Flood, B. Cassarly, C. Sigg, and J. M. Finlan, "Continuous Wide Angle Beam Steering Using Translation of Binary Microlens Arrays and a Liquid Crystal Phased Array," in Computer and Optically Formed Holographic Optics, I. Cindrich and S. H. Lee eds., Proc. SPIE 1211, 296-304 (1990).

6. E. A. Watson, "Analysis of beam steering with decentered microlens arrays," Opt. Eng. 32(11), 2665-2670 (1993).

7. S. Glöckner and R. Göring, "Investigation of statistical variations between lenslets of microlens arrays," Appl. Opt. 36(19), 4438-4445 (1997).

8. P. F. McManamon and E. A. Watson, "Design of a Wide Angle Optical Phased Array Beam Steerer with Limited
Wavelength Dispersion," Proc. IEEE Aerospace Conf., Big Sky, MT, (March 2000).

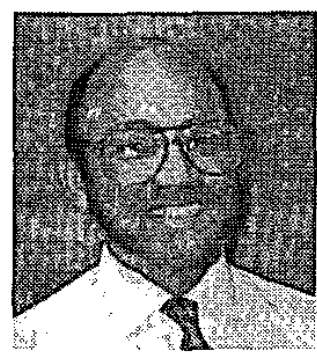

Ed Watson is the Technical Advisor for the Electro-Optical Technology Division, Sensors Directorate, Air Force Research Laboratory. He conducts research in active and passive electro-optic sensors, including the application of dynamic diffractive optical components to the agile steering and shaping of monochromatic and polychromatic light beams, development of multi-phenomenology laser radar imagers, and modeling of aliasing and blurring in sampled imagery. He has a Ph. D. in Optics from the University of Rochester, and an M. S. degree in Optical Sciences and a B.S. degree in Physics from the University of Arizona.

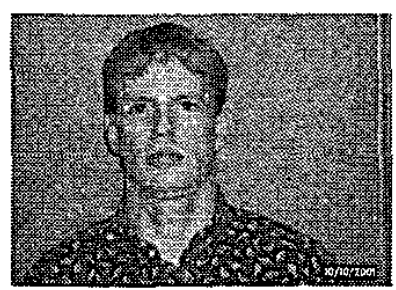

Bert Whitaker is an Engineering Physicist with OptiMetrics, Inc., working under contract with the Multi-Function ElectroOptical Branch of Wright Laboratory at WrightPatterson Air Force Base. His current research involves agile beam steering using microlens arrays. This work includes the development and refinement of basic and novel methods of characterizing such devices and evaluating their performance. His other experimental work dealt with the use of ultra-high vacuum spectroscopy in the growth and characterization of thin films, and the use of liquid crystal displays to generate holographic images. He has a BS degree in Physics, and a $B S E$ degree in Engineering Physics from Wright State University, as well as an MS degree in Physics from Ohio University.

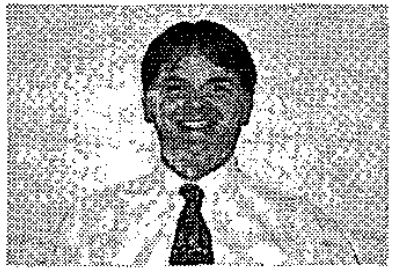

Christopher D. Brewer. Born in West Virginia, Dr. Brewer received a BS degree in physics from Marshall University in 1995. He then went on to obtain a MS degree in 1997, and subsequently his Ph.D.

in 2001, from the University of Dayton in electro-optics while working as a research assistant at Wright Patterson Air Force Base. Currently, he is a member of the University of Dayton Research Institute where he is actively involved with the Electro-Optic Sensors Group of the Air Force Research Laboratory pursuing agile beam steering 
applications of both liquid crystal devices and micro-lens arrays. Other research interests include nonlinear optics and materials, ladar imaging, and glint target tracking.

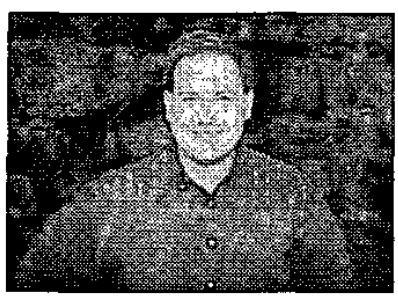

Scott Harris is a research Physicist in the MultiFunction Electro-Optical Technology Branch, Sensors Directorate, Air Force Research Laboratory. Currently the bulk of his research is

focused on the application and characterization of advanced, liquid crystal based, diffractive optical elements. Other research interests include optical diagnostics and spectroscopy, aero-optics, and computational physics. Scott has a Ph.D. in Mechanical and Aerospace Engineering from Princeton University, a M.S. degree in Aerospace Engineering from Stanford University, and a B.S. in Engineering and Applied Science from Caltech. 